\title{
Single Sound Source SLAM
}

\author{
Rodrigo Munguía and Antoni Grau \\ Department of Automatic Control, UPC, \\ c/ Pau Gargallo, 5 E-08028 Barcelona, Spain \\ \{rodrigo.munguia, antoni.grau\} @upc .edu \\ http://webesaii.upc.es
}

\begin{abstract}
Bearing-only Simultaneous Localization and Mapping (SLAM) is a partially observable SLAM problem, in wich the sensor used for perceiving the robot's enviroment, provides only-angular information respect to the landmarks, and therefore does not give enough information to compute the full state of a landmark from a single observation. In this context, vision-based systems have also gained a great interest in the robotics community. Nevertheless the use of "sound sources" as map's features have been very little explored in SLAM. In this work a method for performing SLAM with sound sources is presented. A robot capable of sense bearing information respect to an external sound source with modest angular acuity $\left(-10^{\circ}\right)$ is considered. At the robot trajectory start, the sound source position is unknown; while the robot moves, the position of the sound source and the robot position in a global coordinate frame are both estimated. Experimental results with simulations and with a real robot demonstrate that tracking a unique source sound is enough to reasonably correct the odometry information provided by the encoders.
\end{abstract}

Keywords: SLAM, Sound Sources, Bearing Sensors.

\section{Introduction}

The on-line robot estimation position from measurements of self-mapped features is a class of problem called, in the robotics community, as Simultaneous Localization and Mapping (SLAM) problem, which is one of the fundamental problems in robotics. SLAM consists in incrementally building a consistent map of the environment and, at the same time, localizing the position of the robot while it explores its world.

Nowadays several advances have appeared in this area, specially using range sensor, such as laser and sonar rings, and more recently with cameras as the main sensor. On the other hand the use of the hearing sense in localization and mapping has been much less explored. In this case, sound sources (artificial and natural) are used as landmarks for being included in the robot's map in order to localize it along the time.

Most robot sound localization approaches focused on improving the sensor capabilities (robustness, acuity, etc.) $[1,3,4,5,6,12]$; in these cases the word 'localization' refers to the estimation of the sound source respect to the robot in a robot coordinate frame in order to perform navigation; in that sense, a typical experiment is that a robot autonomously follows a sound source. In this work 'localization' refers to estimating 
the position of the robot along the time in a world coordinate frame. Others methods, [2], combine audio and vision for localizing speakers.

In [7], there is an attempt to localize a mobile robot using several structured sound sources emitting distinctive codes in a similar approach to GPS system, where the position of each sound source is known a priori precisely.

Our work focuses on the inclusion of the hearing sense in SLAM, attempting to localize a robot without a priori information of the sound source location, and at the same time localizing the robot position along the time while it is moving freely in its environment. The experiments presented in this paper consist in moving the robot over a predefined trajectory (for ground truth comparison), while it is capable of tracking the direction of a single sound source, then, the encoders and the bearing information are fused in a SLAM scheme in order to recover the trajectory of the robot. In that sense if the robot is capable to track the direction of the sound source with a relatively modest acuity $\left(-10^{\circ}\right)$, the algorithm is able to reasonably correct the

error propagation of the odometry information provided by the robot's encoders.

The paper is organized as follows: Section II describes the proposed sound-based SLAM algorithm and simulations. Section III gives a brief description of the implemented robot for testing the proposed method. Section IV exposes the experiments realized with the real robot and finally, in section $\mathrm{V}$, conclusions are presented.

\section{Sound-Based SLAM Algorithm}

In this paper the term 'sound sensor' refers to a robot sub-system capable of providing directional acoustic sensing (bearing information) independently on the number of transducers $(2,3$ or a ring of microphones), the acoustic technique (interaural time difference (ITD), interaural phase difference (IPD), interaural intensity difference (IID), etc.) and the kind of sound source (natural or artificial). In that sense a sound sensor has some similarities with monocular vision since in both cases depth information cannot be directly retrieved. A big distant object can appear in an image with the same size of a small close object and a loud distant sound source can be heard similar to a weak close one. In that context some of the principles applied in the monocular-SLAM methods can be used in sound-based SLAM (SSLAM) and part of the algorithm presented below is derived from previous authors' works in monocular-SLAM $[8,9]$.

\subsection{Robot Model}

A differential drive mobile robot with 2-dof equipped with a sound sensor is considered. The robot state $x_{v}$ expressing the vehicle pose (joint of position and orientation) is defined by:

$$
x_{v}=\left[x_{v} y_{v} \theta_{v}\right]^{T}
$$

The robot discrete motion prediction model is:

$$
\left[\begin{array}{c}
x_{v(k+1)} \\
y_{v(k+1)} \\
\theta_{v(k+1)}
\end{array}\right]=\left[\begin{array}{c}
x_{v(k)}+\delta \cos \left(\theta^{\prime}\right) \\
y_{v(k)}+\delta \sin \left(\theta^{\prime}\right) \\
\theta_{v(k)}+\theta^{\prime}
\end{array}\right]
$$


being $\delta$ the distance traveled by the robot's center and $\theta$ ' the turn realized by the robot at each instant $k$ :

$$
\delta=\left(\delta_{\mathrm{r}}+\delta_{\mathrm{l}}\right) / 2, \theta^{\prime}=\left(\delta_{\mathrm{r}}+\delta_{\mathrm{l}}\right) / b
$$

derived from the distance traveled for each robot's wheels $\delta_{\mathrm{r}}, \delta_{1}$ and the separation $b$ between them. Normally $\delta_{\mathrm{r}}, \delta_{\mathrm{l}}$ are obtained from the robot's encoders.

An Extended Kalman Filter (EKF) propagates the robot pose estimates, as well as feature estimates.

\subsection{Features Parameterization}

The complete state that includes the features $y$ is made of:

$$
x=\left[x_{v}^{\mathrm{T}}, y_{1}^{\mathrm{T}}, y_{2}^{\mathrm{T}}, \ldots y_{n}^{\mathrm{T}}\right]^{\mathrm{T}}
$$

where a feature $y$ represents a 2D sound source $i$ defined by the 4-dimension state vector:

$$
y_{i}=\left[x_{i}, y_{i}, \theta_{i}, \rho_{i}\right]^{\mathrm{T}}
$$

which models the $2 \mathrm{D}$ point located at (Figure $1 \mathrm{~b}$ ):

$$
\left[\begin{array}{l}
x_{i} \\
y_{i}
\end{array}\right]+\frac{1}{\rho_{i}} m\left(\theta_{i}\right)
$$

where $x_{i}, y_{i}$ are the robot center coordinates when the feature was observed the first time; and $\theta_{\mathrm{i}}$ represent azimuth (respect to the world reference $W$ ) for the directional vector $m\left(\theta_{\mathrm{i}}\right)$. The point depth $d_{i}$ is coded by its inverse $\rho_{i}=1 / d_{i}$.

In [10] and [11] is shown that the used of an inverse depth parametrization for bearing-only SLAM can improve the linearity of the measurement equation even for small changes in the sensor position converted in small changes in the parallax angle, this fact allows a Gaussian distribution to cover uncertainty in depth which spans a depth range from nearby to infinity. It is well known the relevance of a good uncertainty Gaussian representation in a scheme based in EKF. Figure 1(a) shows a simulation of a low parallax $\alpha\left(0.5^{\circ}\right)$ point reconstruction when it is captured by the sensor at known locations.

The different locations of the robot, along with the location of the already mapped features, are used to predict the feature angle $h_{i}^{z}$ (angle describing the direction of the incoming sound) in the robot coordinate frame.

$$
h_{i}^{z}=\arctan 2\left(\frac{1}{\rho_{i}} \sin \left(\theta_{i}\right)+y_{i}-y_{v}, \frac{1}{\rho_{i}} \cos \left(\theta_{i}\right)+x_{i}-x_{v}\right)-\theta_{v}
$$

At this stage it is assumed that the sound sensor is capable of tracking and discriminating between sound sources (a hard challenge in a real implementation, indeed). 


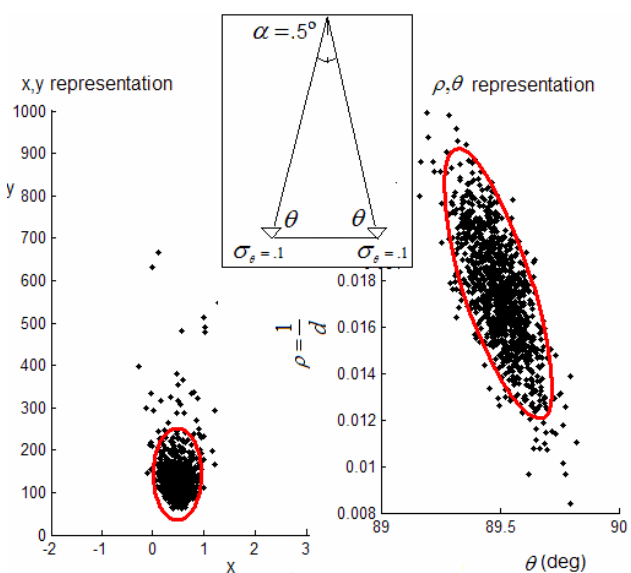

(a)

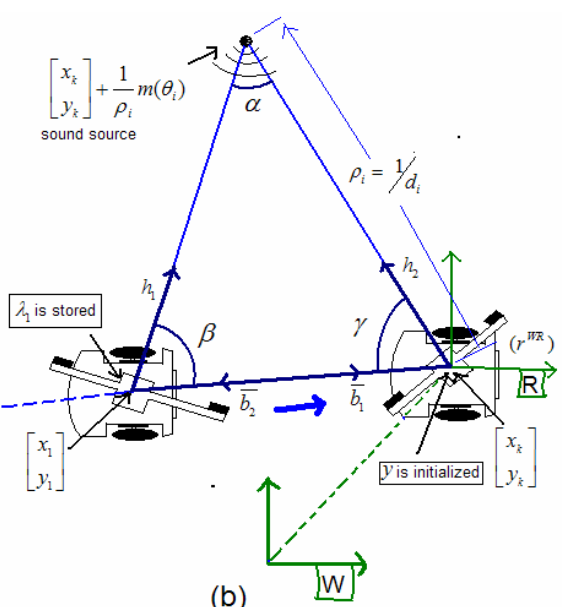

(b)

Fig. 1. (a) Simulation of a point reconstruction from two observations with a low parallax. It is shown how the reconstruction error coded in c is Gaussian, while if it is coded as cartesian $X Z$ is not Gaussian. Red ellipses represent linear uncertainty propagation. (b) Feature parametrization and initialization.

\subsection{Features Initialization}

A sound sensor estimating the bearing of sound sources is considered; therefore depth information cannot be obtained in a single measurement. To infer the depth of a feature, the robot must observe it repeatedly as it freely moves through its environment, each time estimating the angle from the feature to its center. The difference between the angles measurements is the feature parallax. Actually, parallax is the key that allows to estimating features depth. In the case of indoor sequences, centimeters are enough to produce parallax, on the other hand, the more distant the feature, the more the robot has to travel to produce parallax.

Features need an especial treatment before to be added to the stochastic map. In this case a delayed feature initialization, proposed in previous works [8, 9], is used. For a wide explanation in the delayed feature initialization method, please see these references.

\subsection{Algorithm Simulations}

Figure 2 shows the simulation of the algorithm for a circular trajectory, a Gaussian noise with a variance of 0.01 is added to the odometry (typical in encoders based systems). For the sound sensor, a variance $\sigma=5^{\circ}$ is considered. In the simulation it is supposed that the sound sensor is able to tracking the sound source over the $360^{\circ}$; in the case of having several sound sources it is supposed that the sensor can do a single source measurement at a time and the separation of sound sources (data association problem) is obviated.

In the case of a single sound source the correct position initialization of the feature (sound source) in the system plays a very important role; if the feature is initialized of its real position even the filter could diverge, if the single feature is initialized properly the trajectory can be recovered acceptably. 

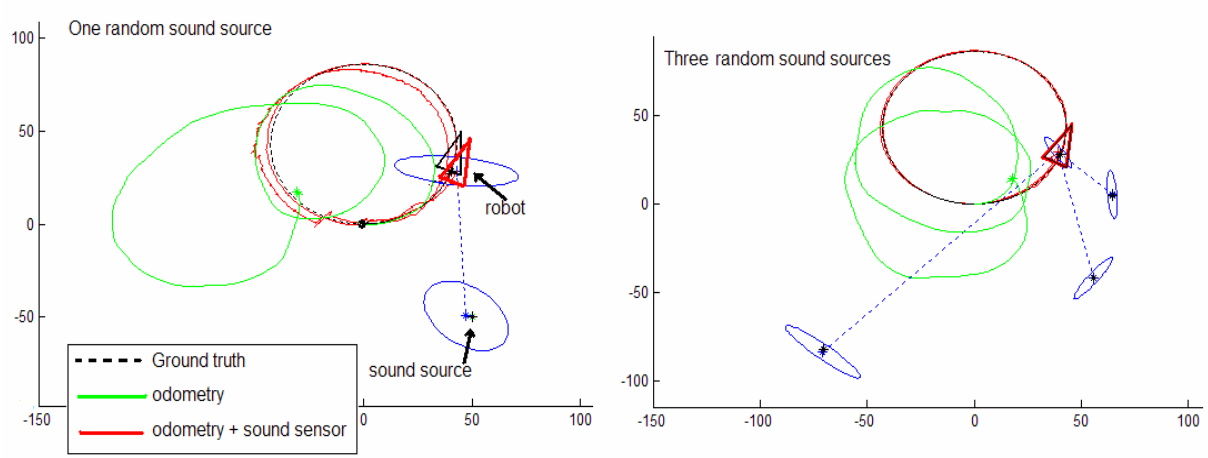

Fig. 2. Simulation of the algorithm with initial random sound sources positions: Upper plot shows an example with a single sound source. Lower plot shows an example with three sound sources. Blue ellipses represent robot and sound sources uncertainties positions with $2 \sigma$. Note that with three sources the trajectory and its positions are more precisely recovered whereas the uncertainties are lower.

In the case of several sound sources the robustness of the algorithm increases, with a lower dependence on the initialization values; robot position and sources positions uncertainties are lower due to an increase of available information in the system.

\section{Robot Implementation}

Observing the simulations results it is clear that the algorithm works better with more than one sound source, nevertheless in a real situation it is obvious that to track and to discriminate among several sound sources is a very challenging effort. There are several proposals in this approach but they are out of the scope for the main objective of this work. On the other hand, observing simulation results, it is surprising to note that a single sound source is enough for correct the odometry error in an acceptable manner, this fact has motivated the authors to testing the algorithm in a real robot equipped with a sound sensor of uncomplicated implementation.

Figure 3 (right) shows the implemented robot for the experiments; it is equipped with optical encoders for odometry, sensors for tracking floor lines, a radio modem for communication with a $\mathrm{PC}$ and a $360^{\circ}$ rotating head capable of tracking the sound direction of a single ultrasonic source.

For implementing the sound sensor (left), a $360^{\circ}$ sound-servo configuration is used. In order to avoid the sound source separation issue, a structured ultrasonic source is used. This sound source emits eight $40 \mathrm{kHz}$ pulses every 40 milliseconds. Two ultrasonic transceivers are mounted at the ends of a $360^{\circ}$ rotating head with a separation of $25 \mathrm{~cm}$ between them. The head is driven by a modified servo for continuous rotation. A microcontroller measures the interaural time difference (ITD) in the ultrasonic receivers, the ITD is used as a feedback signal in a PI controller that drives the servo looking for an ITD close to zero. The rotation head is connected by gears to a continuous rotation potentiometer for generating (with a voltage divisor) a voltage proportional to the bearing of sound source. The potentiometer has a wedge of $30^{\circ}$ 


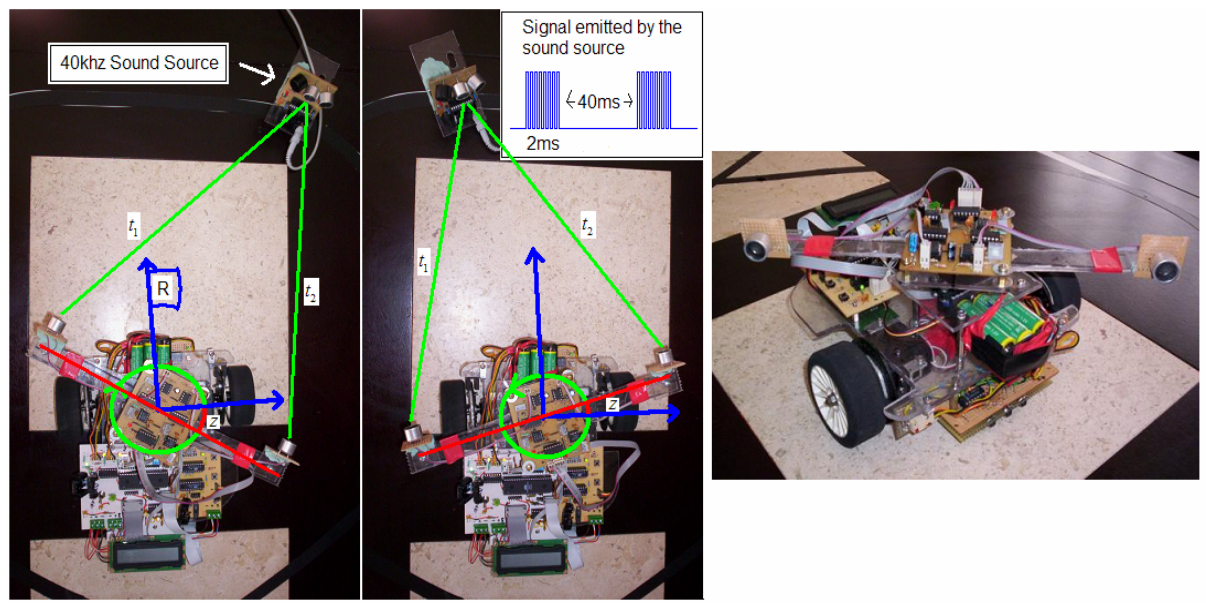

Fig. 3. Interaural time difference (ITD) is used as feedback signal in a $360^{\circ}$ servo configuration for implementing the robot's sound sensor (left). Picture of the robot used for testing the algorithm; the robot is implemented by the authors specifically for the experiments of this paper. Note the ultrasonic receivers at the ends of the rotating head (right).

where is not possible to obtain measurements, therefore the sound sensor has a working range of $330^{\circ}$. Finally this voltage is converted to a binary value by an ADC. This sound sensor has acuity around $-10^{\circ}$.

\section{Experiments with Real Data}

For the experiments with real data, the robot tries to track some predefined paths (figure $4 \mathrm{~h}$ ); each time that the control program in the $\mathrm{PC}$ requests a data, the current encoders' counts (both wheels) and the ADC value are sent by radio modem to the PC. For every request these three values are stored in a table, after several turns to the circuit the data table is used as the input for the SSLAM MatLab implementation, it is important to say that is feasible to implement the algorithm in a real time version.

Figure 4 illustrates the results obtained with the data captured from the robot's sensors, the upper plots ( $a, b, c, d$ and e) show the process for initializing the sound source in the robot's map; only when a minimum parallax value is reached the new feature is added to the stochastic map. Lower plots ( $\mathrm{f}$ and $\mathrm{g}$ ) show the results for two different trajectories, an oval path (f) and an eight-shape path (g). In plot (f) note how after 6 laps (around $24 \mathrm{~m}$ of travel) the trajectory is reasonably well recovered with the SSLAM algorithm (red trajectory) compared with the estimates using only odometry (green). In (g) a more challenging trajectory is followed by the robot, note how the big drift showed by the robot's odometry is well bounded in the trajectory estimated by the SSLAM algorithm.

The non-operative wedge of $30^{\circ}$ in the robot's head has little effect in the estimated trajectory; maybe in plot (f) can be slightly appreciated, but the trajectory is rapidly recovered by the algorithm when the head enters in the fully operative area of $330^{\circ}$. 

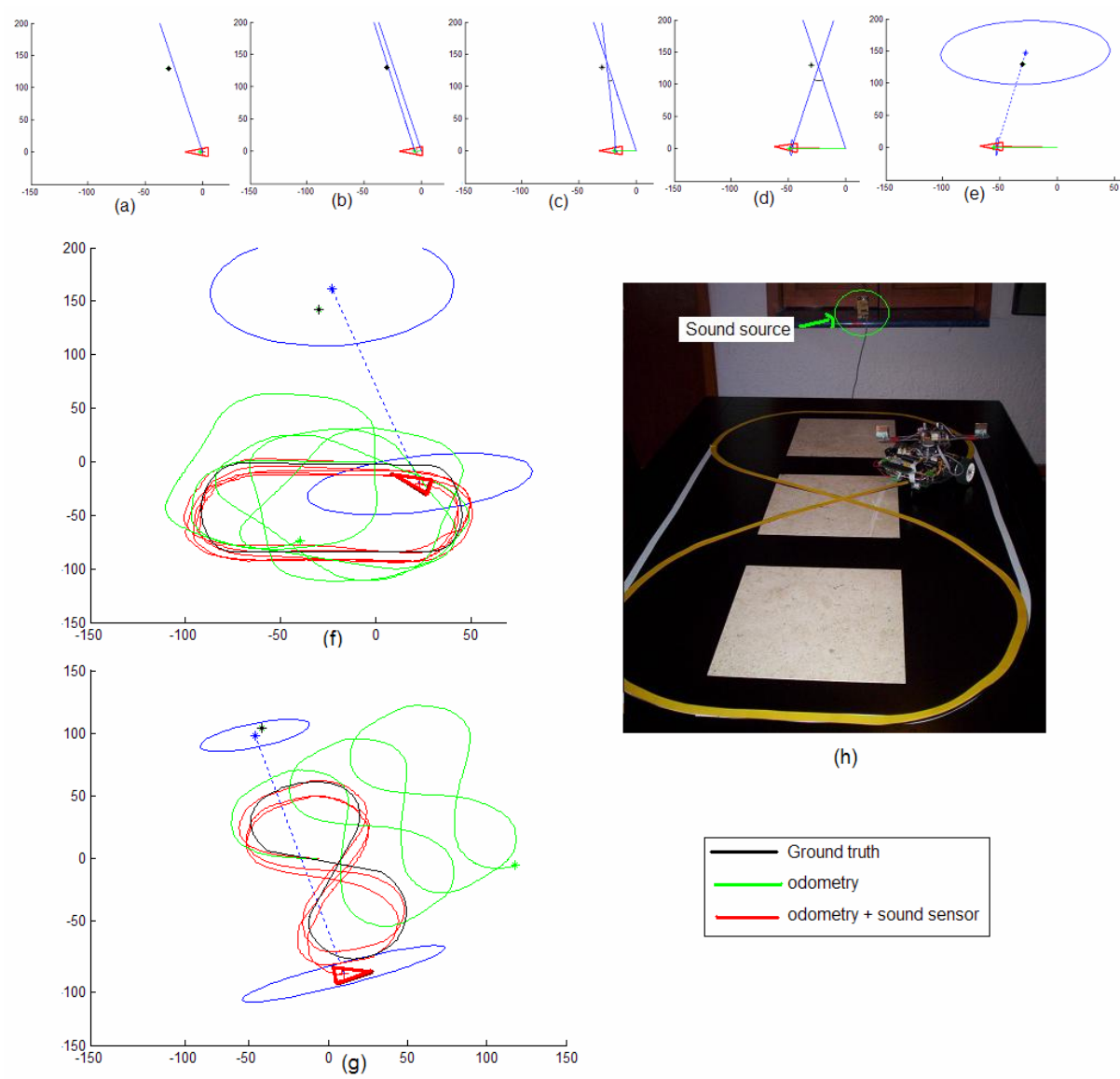

(h)

Fig. 4. Experiments with real data taken from the robot's sensors are realized. Upper plots $(a, b, c, d, e)$ illustrate the initialization of a sound source in the robot's map as it is described in section II.C; when the feature is detected for the first time (a) some part of the state and measurement are stored, this information is used in subsequent steps (for example in b,c,d) together with the current state and measurements to evaluate the parallax, when the parallax is bigger than some threshold the sound source is initialized as a new feature in the stochastic map (e). Lower plots (f,g) show the estimates for two different predefined trajectories followed by the robot: an oval (f) and an eight-shape (g), in both cases note how the estimated trajectories with the sound based SLAM (red) are reasonably recovered after several laps, on the other hand note the big error propagation in the estimated trajectories with only the robot's odometry (green).

\section{Conclusions}

In this work a novel algorithm, called SSLAM, has been presented. This algorithm performs sound-based simultaneous localization and mapping. A robot subsystem (in this work, sound sensor), capable of providing bearing information respect to one or several sound sources, is closely related (from the algorithmic point of view) with a 
camera, in that sense a monocular SLAM method proposed in previous authors' works as been modified for working in a sound-based context.

The algorithm simulations show that several sound sources improve the robustness and effectiveness of the method; nevertheless a real sound sensor capable of tracking and separating several sound sources requires a challenging implementation. In that sense, a small mobile robot, capable of tracking a single sound source, is implemented in order to test SSLAM with real data sensor. The experimental results show how the method is able to estimate the sound source position without prior knowledge of the environment; this information is subsequently used for estimating reasonably well the robot's trajectory. It can be appreciated how the error propagation of the encodersbased odometry is bounded.

These experimental results are very promising since the method is tested in a robot with very limited capabilities. The method could be applied in a robot equipped with a more complex sound sensor capable of measuring several natural audio sources.

Acknowledgements. This research was conducted at the Automatic Control Dept of the Universitat Politecnica de Catalunya. It was partially supported by Consolider Ingenio 2010, project CSD2007-00018, CICYT project DPI2007-61452, and IST045062 of the European Community Union

\section{References}

1. Valin, J., Michaud, F., Rouat, J., Lètourneau, D.: Robust sound source localization using a microphone array on a mobile robot. In: International Conference on Intelligent Robots and Systems (2003)

2. Nakadai, K., Okuno, H., Kitano, H.: Realtime sound source localization and separation for robot audition. In: IEEE International Conference on Spoken Language Processing (2002)

3. Murray, J., Erwin, H., Wermter, S.: Auditory Robotic Tracking of Sound Sources using Hybrid Cross-Correlation and Recurrent Network. In: International Conference on Intelligent Robots and Systems (2005)

4. Amir, A., Handzel, B., Andersson, M., Krishnaprasad, P.S.: A Biomimetic Apparatus for Sound-source Localization. In: Proc. IEEE Conference on Decision and Control (2003)

5. Handzel, B., Shah, A.A., Krishnaprasad, V.: Robot phonotaxis with dynamic sound-source localization. In: IEEE International Conference on Robotics and Automation (2004)

6. Mumolo, E., Nolich, M., Vercelli, G.: Algorithms for acoustic localization based on microphone array in service robotics. In: Robotics and Autonomous Systems (2003)

7. Ureña, J., Hernández, A., Jiménez, A.: Advanced Sensorial System for an Acoustic LPS. Journal of Microprocessors and Microsystems (2006)

8. Munguia, R., Grau, A.: Delayed Feature Initialization for Inverse Depth Monocular SLAM. In: European Conference on Mobile Robots (2007)

9. Munguia, R., Grau, A.: Delayed Inverse Depth Monocular SLAM. In: 17th IFAC World Congress (2008)

10. Montiel, J.M.M., Civera, J., Davison, A.: Unified Inverse Depth Parametrization for Monocular SLAM. In: Robotics: Science and Systems Conference (2006)

11. Eade, E., Drummond, T.: Scalable monocular SLAM. In: IEEE Conference on Computer Vision and Pattern Recognition (2006)

12. Czyzewski, A.: Automatic identification of sound source position employing neural networks and rough sets. Pattern Recognition Letters 24 (2003) 